\title{
Incidencia de otitis media aguda en niños del sector Sur Oriente de Santiago antes y después de la implementación de vacuna antineumocócica 10 valente en el programa nacional de inmunizaciones
}

\author{
Incidence of acute otitis media in children from South East area of Santiago \\ before and after the implementation of 10 -valent pneumococcal vaccine \\ in the national immunization program
}

\author{
Macarena Rosenblut Sํㅗ
}

\section{RESUMEN}

Introducción: Streptococus pneumoniae es la principal causa de otitis media aguda (OMA). La vacuna antineumocócica 10 valente (PCV-10) fue introducida al Programa Nacional de Inmunizaciones (PNI) el año 2011. No existen estudios que midan la incidencia de infecciones por $S$. pneumoniae desde la implementación de esta vacuna en el PNI.

Objetivo: Determinar la incidencia de OMA en niños <24 meses que consulten en el Hospital Dr. Sótero del Río (HSR) de Santiago antes y después de la implementación de PCV-10 en el PNI.

Material y método: Estudio retrospectivo, observacional, en HSR entre 2007-2012. Se estudiaron niños <24 meses con diagnóstico de egreso de OMA atendidos en el Servicio de Urgencia (SU) del HSR. Se calculó incidencia de OMA en períodos pre y posvacuna y se compararon ambos períodos.

Resultados: El promedio de niños <24 meses con diagnóstico de OMA por año en el período pre y posvacuna fue de 290 versus $239(P>0,05)$ con un número de consultas totales anuales en niños de la misma edad que se mantuvo constante en el tiempo. La incidencia de OMA en el periodo prevacuna fue de 0,94\% y en periodo posvacuna de $0,76 \%, P>0,05$.

Conclusiones: Nuestro estudio no mostró una diferencia en la incidencia de OMA en niños <24 meses que consultaron en el Servicio de Urgencia (SU) del Hospital Sótero del Río (HSR) antes y después de la implementación de vacuna antineumocócica en el PNI de Chile. Se requiere mantener la vigilancia y una evaluación a más largo plazo para evaluar el real efecto que esta vacuna podría tener en esta patología.

Palabras claves: Otitis media aguda, vacuna antineumocócica.

\footnotetext{
${ }^{1}$ Interna de Medicina, Universidad del Desarrollo.

${ }^{2}$ Médico Otorrinolaringología, Pontificia Universidad Católica de Chile.

${ }^{3}$ Médico Otorrinolaringólogo, Servicio Otorrinolaringología, Hospital Dr. Sótero del Río.
} 


\section{ABSTRACT}

Introduction: Streptococcus pneumoniae is the leading cause of acute otitis media (AOM). The 10-valent pneumococcal vaccine (PCV-10) was introduced into the national immunization program (NIP) in 2011. There are no studies that measure the incidence of infections due to $S$. pneumonie from the implementation of this vaccine in the NIP.

Aim: To determine the incidence of AOM in children <24 months attending the Sótero del Rio Hospital (HSR) of Santiago, Chile, before and after the implementation of PCV-10 in the NIP.

Material and method: Retrospective, observational study in HSR, between 20072012. Children <24 months with discharge diagnosis of AOM treated at the Emergency Room (ER) of the HSR were included. We compared the incidence of AOM in pre and post vaccine periods.

Results: Retrospective, observational study in HSR, between 2007-2012. Children <24 months with discharge diagnosis of AOM treated at the Emergency Room (ER) of the HSR were included. We compared the incidence of AOM in pre and post vaccine periods.

Conclusions: Our study showed no difference in the incidence of AOM in children <24 months who visited the ER of SDR Hospital before and after implementation of pneumococcal vaccine in the NIP in Chile. Prospective surveillance is required to assess the longer term effect of the implementation of this vaccine in the incidence of AOM.

Key words: Acute otitis media, pneumococcal vaccine.

\section{INTRODUCCIÓN}

La otitis media aguda (OMA) es una de las enfermedades infecciosas de etiología bacteriana más frecuente en la población pediátrica ${ }^{1}$, especialmente en menores de dos años, siendo la principal causa de prescripción de antimicrobianos a esta edad²-4. En el año 2009, se estimó una carga de enfermedad en América Latina y el Caribe de 980.000-1.500.000 casos $^{5}$.

Estudios etiológicos a nivel mundial han demostrado que Streptococus pneumoniae es la bacteria más frecuentemente aislada como causa de $O \mathrm{MA}^{6-8}$. Un estudio microbiológico de líquido del oído medio realizado en niños con OMA del sector Sur Oriente de Santiago, Chile, el año 2006, demostró presencia de $S$ pneumoniae en $40 \%$ de los casos, Haemophilus influenzae en $29 \%$, Moraxella catharralis en $7 \%$ y Streptococcus pyogenes en $4 \% 9$.

Actualmente existen 3 vacunas conjugadas antineumocócicas, 7-valente (PCV-7), 10 valente (PCV-10) y 13-valente (PCV-13), todas cuentan con ensayos clínicos donde se ha demostrado su eficacia frente a infecciones invasoras, demostrando además inmunogenicidad y seguridad ${ }^{10-15}$. La vacuna antineumocócica 10 valente tiene cobertura contra 10 serotipos (1-3-4-5-6B-7F-9V-14-18C19F y 23F), fue aprobada en Chile el año 2009 e incorporada al Programa Nacional de Inmunizaciones (PNI) desde el 1 de enero de 2011 en un esquema de 3 dosis, a los 2, 4 y 12 meses $^{16}$. La proteína transportadora de esta vacuna es la proteína $\mathrm{D}$ de $\mathrm{H}$ influenzae no tipificable (Hi-NT), lipoproteína de superficie de $42 \mathrm{Kd} 17$ que se une a cada uno de los serotipos presentes en la vacuna. Esta proteína está presente en $H$ influenzae tanto capsulado como no tipificable, y se postula su potencial para proteger ante cualquier infección por esta bacteria ${ }^{18}$.

La eficacia de la vacuna antineumocócica conjugada 10 valente frente a OMA fue probada en un estudio realizado en la Republica Checa el año 2006, donde 4.968 niños de 3 meses a 36 meses fueron distribuidos aleatoriamente para recibir vacuna antineumocócica 0 inmunización contra hepatitis A. En este estudio, se demostró que la incidencia de OMA en el grupo que recibió vacuna antineumocócica disminuyó en 33,6\%, reduciéndose en 52,6\% los episodios de OMA por serotipos de $S$ pneumoniae presentes en la vacuna. El estudio mostró además una disminución de 35,5\% de las OMA causadas por Hi-NT'18. 
En Chile, las OMA por $S$ pneumoniae están causadas principalmente por 4 serotipos, que concentran el $50 \%$ de las infecciones: 19F, 14, 23F y 56,9. Estos 4 serotipos son cubiertos por PCV-10. En nuestra población, PCV-7 tendría cobertura teórica para el $50 \%$ de los serotipos causantes de OMA, PCV-10 para el $63 \%$ y PCV-13 para el $67 \%$, además de la eventual protección de PCV-10 frente a OMA causadas por Hi-NT. La protección de esta vacuna no es de $100 \%$ de los serotipos vaccinales sino que se estima en una protección real cercana a $57 \%$ para las OMA causadas por $S$ pneumoniae, 35\% para las OMA por $H$ influenzae y $65 \%$ de protección cruzada para los serotipos no vaccinales de $S$ pneumoniae ${ }^{18}$.

De acuerdo a dos estudios etiológicos de OMA realizados en niños en Chile, que entregan información sobre los serotipos de $S$ pneumoniae involucrados en esta patología y a los antecedentes de protección teórica conocidos para PCV-10, la eficacia protectora de PCV-10 en niños en Chile frente a OMA se aproximaría a $28 \% 6,9$.

\section{OBJETIVO}

El objetivo de este estudio fue evaluar la incidencia de OMA en niños <24 meses del sector Sur Oriente de Santiago antes y después de la implementación de vacuna antineumocócica 10 valente en el PNI.

\section{MATERIAL Y MÉTODO}

Estudio retrospectivo, observacional, realizado en el Hospital Dr. Sótero del Rio (HSR). Se estudió el número de niños $<24$ meses con diagnóstico de egreso de OMA que consultaron en el Servicio de Urgencia (SU) del HSR desde el 1 de enero de 2007 al 31 de diciembre de 2012 y se obtuvo el dato de las consultas totales al SU del HSR en niños $<24$ meses por año en el mismo período de tiempo.

Se dividió la observación en dos períodos de tiempo: Prevacuna: $(2007,2008,2009,2010)$ y posvacuna: (2012). Se calculó la incidencia de OMA en el período pre y posvacuna y se realizó una comparación entre ambos períodos. El año 2011 fue considerado un año de transición (año de inicio de vacuna PCV-10 en el PNI de Chile) por lo que fue considerado en la descripción general de la población pero no en los cálculos comparativos de los períodos pre y posvacuna.

Los datos de número total de consultas y de consultas por OMA al SU del HSR fueron obtenidos desde el Servicio de Estadística del Hospital. Los niños con diagnóstico de egreso de OMA desde el SU se identificaron según código CIE-10: H.65 Otitis media no supurativa, H.66 Otitis media supurativa y otitis media sin especificar y H.67 Otitis media en enfermedades clasificadas en otro código.

\section{Análisis estadístico}

Se compararon los datos de los casos totales del período 2007-2010 con los del año 2012, evaluando si existía una disminución significativa de casos de OMA en el SU del HSR. Para la comparación se utilizó prueba de Chi-cuadrado. Se consideró significativo un valor de $\mathrm{P}<0,05$.

\section{RESULTADOS}

\section{Casos de OMA por año en relación a consultas por año en niños <24 meses en el SU Hospital Dr. Sótero del Río}

Durante los 6 años del período de estudio (2007-2012), hubo un promedio de 31.187 (29.358-33.178) consultas al año al SU del HSR en niños <24 meses (Figura 1), número de consultas que no mostró variación significativa durante los años analizados $(P>0,05)$.

Del total de consultas por año en niños <24 meses, un promedio de 271 (223-387) correspondió a niños con diagnóstico de egreso de OMA, lo que otorga una incidencia promedio de $0,86 \%$ por año. La Figura 2 muestra la incidencia de OMA en el grupo etario estudiado por año, con valores que no mostraron variación estadísticamente significativa durante el periodo de estudio $(P>0,05)$.

\section{Incidencia de OMA en períodos pre y posvacuna}

El promedio de niños $<24$ meses con diagnóstico de OMA en el período pre y posvacuna fue de 290 versus 239 respectivamente $(P>0,05)$. La incidencia de OMA en niños <24 meses en el período prevacuna fue de $0,94 \%$ y en el período posvacuna fue de $0,76 \%$ (Figura 3 ). 


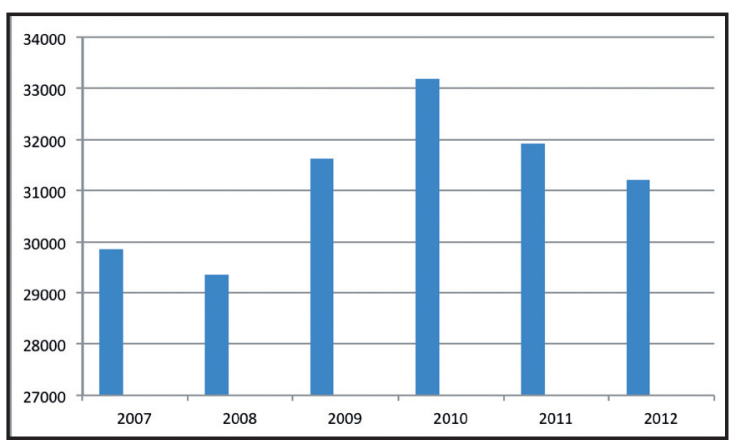

Figura 1. Número de consultas anuales al Servicio de Urgencia del Hospital Dr Sótero del Río en niños <24 meses de edad entre los años 2007 y 2012.

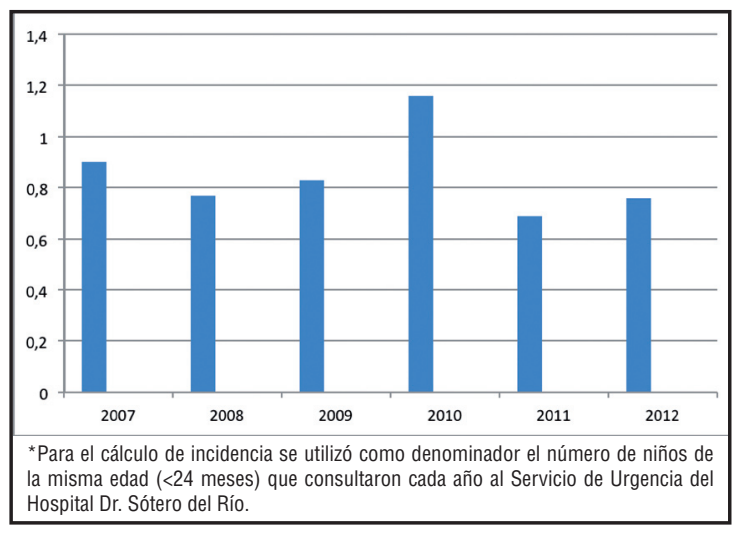

Figura 2. Incidencia de otitis media aguda por año en $<24$ meses* $^{*}$ Período 2007-2012.

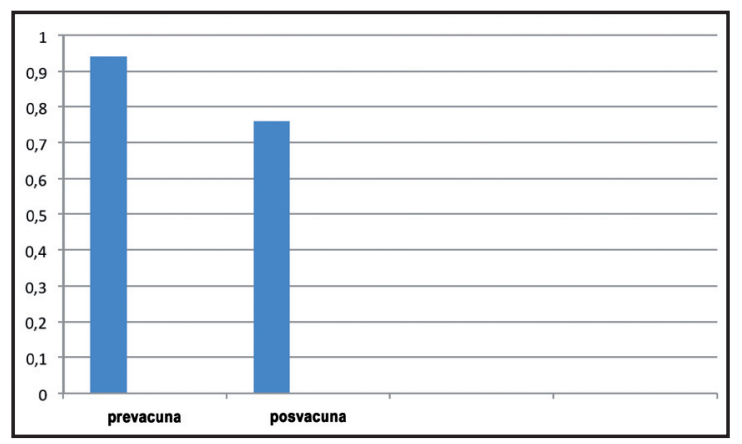

Figura 3. Incidencia de OMA en el periodo prevacuna (2007-2010) y posvacuna (2012) en niños $<24$ meses.

\section{DISCUSIÓN}

Nuestro estudio no logró mostrar diferencias significativas en la incidencia de OMA en la población estudiada en los periodos pre y posvacuna. Si bien se observó una tendencia a la disminución de la incidencia de OMA en el periodo posvacuna (2012) de 0,16\%, ésta no alcanza significancia estadística $(p>0,05)$.

Es conocido que el error diagnóstico de OMA en los SU es de aproximadamente $50 \%{ }^{6}$, generalmente hacia un sobrediagnóstico, 10 que podría dificultar la medición del efecto de la vacuna en la incidencia de OMA. Sin embargo, este error diagnóstico es estable en el tiempo, lo que fue demostrado previamente en dos estudios etiológicos de OMA realizados en el HSR, en que casos de presunta OMA diagnosticados en el SU eran derivados al Servicio de Otorrinolaringología para certificación diagnóstica y extracción de líquido desde oído medio para diagnóstico etiológico. El año 2001, el error diagnóstico fue de 58\% (222/529 niños con presunto diagnóstico de OMA tenían efectivamente esta patología $)^{6}$ y el 2010 , el error diagnóstico fue de $57,5 \%$ (136/320) (Rosenblut A, datos no publicados). De acuerdo a este error diagnóstico, que podría ser lo que ocurre en el manejo cotidiano de los niños con OMA, el eventual efecto de una vacuna antineumocócica en esta patología tendría que estimarse solo sobre el $50 \%$ de las OMA que están bien diagnosticadas, considerando este antecedente podríamos suponer que la diminución del 16\%, correspondería en realidad a una disminución real cercana al doble (ya que el efecto protector de la vacuna sólo se expresaría en las OMA reales), la que coincide con la esperada en forma teórica lo que es un factor más a considerar en este complejo problema.

Nuestros resultados son preliminares, el efecto de la implementación de vacuna antineumocócica en el PNI de Chile sobre la incidencia de OMA requiere una evaluación a más largo plazo, que implique una vigilancia activa y prospectiva de los casos de OMA en los siguientes años, o idealmente, se haga un estudio de evaluación de incidencia de OMA y etiología a través de punción timpánica, donde se evalúe además el rol relativo de $S$ pneumoniae en OMA en una era de vacunación antineumocóccica y la representación relativa de cada serotipo en el mismo escenario. 


\section{Bibliografía}

1. Kathy C, Mathew W. Otitis media, ear nose and throat. eMedicine 2005; 1-11.

2. Berman S. Otitis media in developing countries. Pediatrics 1995; 96: 126-31.

3. Block SL, Harrison CJ, Hedrick J, et al. Penicilinresistant Streptococcus pneumoniae in acute otitis media: risk factor, susceptibility patterns and antimicrobial management. Pediatr Infec Dis J 1995; 14: 751-9.

4. Dowel SF, Schwartz B. Resistant pneumococci: protecting patients through judicious use of antibiotics. Am Fam Physician 1997; 55: 164754.

5. Valenzuela Mt, O`Loughlin R, De la Hoz F, et al. The burden of pneumococcal disease among Latin American and Caribbean children: review off the evidence. Rev Panam Salud Pública 2009; 25: 270-9.

6. Rosenblut A, Santolaya ME, González P, et al. Bacterial and viral etiology of acute otitis media in chilean children. Ped Infect Dis J 2001; 20: 501-7.

7. Bluestone C. Terminology and classification. In: Bluesstone C, editor. Evidence based otitis media. Hamilton: Saint Louis 1999: 85-103.

8. Leibovitz E, Jacob MR, Dagan R. Haemophilus influenzae: a significant pathogen in acute otitis media. Ped Infect Dis J 2004; 23: 1142-52.

9. Rosenblut A, Santolaya ME, González P, et AL. Penicillin resistance is not extrapolable to amoxicillin resistance in Streptococcus pneumoniae isolated from middle ear fluid in Children with acute otitis media. Annals of Otol, Rhinol \& Laringol 2006; 115: 186-90.

10. Black S, Shinefield H, Fireman B, et al. Efficacy, safety and inmunogenecity of heptavalen pneumococcal conjúgate vaccine in children. Northern California Kaiser Permanente Vaccine Study Center Group. Ped Infect Dis J 2000; 19: 187-95.

11. Vesikari T, Wysocki J, Chevallier B, et al. Immunogenicity of the 10-valent pneumococcal nontypeable Haemophilus influenzae Protein D Conjugate Vaccine compared to the licensed
7Vcrm Vaccine. Ped Infect Dis J 2009; 28 : S66-S76.

12. Ruiz-Palacios GM, Guerrero ML, HernándezDELGADo L, ET AL. Immunogenicity, reactogenicity and safety of the 10-valent pneumococcal nontypeable Haemophilus influenzae protein D conjugate vaccine in Mexican infants. Hum Vaccin 2011; 7: 1012-8.

13. Van den Bergh MR, Spijkerman J, Francois N, et AL. Immunogenicity, safety, and reactogeniciy of the 10-valent pneumococcal nontypeable Haemophilus influenzae protein $D$ conjugate vaccine and DTPa-IPV -Hi B co administered as a 3-dose primary vaccination schedule in the Netherlands: a randomized controlled trial. Ped Infect Dis J 2011; 30: 70-8.

14. Center for Disease Control and Prevention (CDC) Licensure of a 13-Valent Pneumococcal Conjugate Vacinne (PCV13) and recommendations for use among children-advisory committee on immunization Practices (ACIP), 2010. http.// www.cdc.gov/mmwr/preview/mmwrhtml/ mm5909a2.htm.

15. Bryant Ka, Block SL, Baker SA, et al. PCV13 Infant Study Group. Safety and immunogenicity of a 13-valent pneumococcal conjugate vaccine. Pediatrics 2010; 125: 866-75.

16. Instituto de Salud Pública de Chile. Ficha de productos registrados.-Synflorix vacuna conjugada y adsorbida de polisacárido neumococcico y proteína $\mathrm{D}$ de Haemophilus influenzae no tipificable (NTHi), suspensión inyectable. Accessed on May 7TH on http://200.68.11.21/RegistrosISP/ fiFichaProducto.asp?RegistrolSP=B2077/09.

17. Poolman JT, Bakaletz L, Cripps A, et al. Developing a nontypeable, Haemophilus influenzae (NTHi) vaccine. Vaccine 2000; 19 (suppl 1): S108-15.

18. Prymula R, Peeters P, Chrobok V et al. Pneumococcal capsular polysaccharides conjugated to protein $\mathrm{D}$ for prevention of acute otitis media caused by both Streptococcus pneumoniae and non-typable Haemophilus influenzae: a randomized double-blind efficacy study, Lancet 2006; 367: 740-8.

\footnotetext{
Dirección: Andrés Rosenblut

Atalaya 11152, Las Condes, Santiago

E mail: arosenblut@sochiorl.cl
} 